\title{
Studies on Photochemical Behavior of Amphiphilic Polymers Consisting of Styrenesulfonate, Butyl Methacrylate, and Vinylcarbazole Probe in Aqueous and Nonaqueous Solutions
}

\author{
Tokuji MiYashita, ${ }^{\dagger}$ Shinji TakeuCHI, ${ }^{*}$ and Minoru Matsuda* \\ Department of Biochemistry and Engineering, Tohoku University, \\ Aoba Aramaki, Aoba-ku, Sendai 980, Japan \\ * Chemical Research Institute of Nonaqueous Solutions, Tohoku University, \\ Katahira 2-1-1, Aoba-ku, Sendai 980, Japan
}

(Received April 1, 1989)

\begin{abstract}
An amphiphilic polymer consisting of $p$-styrenesulfonate (SS), butyl methacrylate (BMA), and a small amount of vinylcarbazole $(\mathrm{VCz})$ was prepared. The fluorescence spectra from the carbazole chromophore covalently attached to the polymer as an emission probe showed a monomer fluorescence and no excimer emission. Whereas the shape and the wavelength of the monomer fluorescence were unchanged with solvents, the intensity varied with the solvents. The fluorescence was most intense in water where the polymers form a latex particle. The intensity was correlated with the solubility parameter of the solvents; it decreased with this parameter in organic solvents. In good solvents for the polymer such as tetrahydrofuran (THF), the expanded form of the polymer chain allows the energy migration between the carbazole units. Then the migration energy is trapped by an energy trap site existing on the polymer chain, resulting in a decrease in intensity. The quenching of the photoexcited carbazole by organic cations was also studied from measurements of the steady-state emission intensity. The quenching behavior in water was quite different from those in THF and dimethylformamide (DMF). In water, the quenching became more effective in more dilute polymer concentration. The incorporation of the quencher due to a hydrophobic and/or electrostatic interactions into the polymer chain promoted the quenching.

KEY WORDS Amphiphilic Polymer/Emission Probe / Electron Transfer /

Molecular Assembly /
\end{abstract}

The photochemistry in molecular assembly systems has been extensively investigated. Especially photoinduced electron transfer process in microheterogeneous environments with charged interfaces such as micelle, ${ }^{1}$ microemulsion, ${ }^{2}$ vesicle ${ }^{3}$ polyelectrolyte, ${ }^{4}$ and LB multilayer ${ }^{5}$ is now receiving much attention in relation to solar energy conversion. It is necessary to design various molecular assemblies for a desired functionality. To this end, polymer systems are convenient because the monomers with a varied property can be intro- duced as a comonomer. Amphiphilic polymers consisting of a hydrophobic and a hydrophilic monomer unit can yield a variety of microheterogeneous environment by change in copolymer compositions. ${ }^{6}$ Moreover, the third monomer having photoactive properties has been often introduced by terpolymerization to prepare a photoredox reactive site or a probe molecule.

In this study, we prepared the amphiphilic polymer consisting of $p$-styrenesulfonate, butylmethacrylate, and a small amount of vinyl-

\footnotetext{
$\dagger$ To whom correspondence should be addressed.
} 
carbazole (emission probe). Through measurement of the fluorescence spectra and quenching reaction of the emission probe in various solutions, we investigated the properties and behavior of the amphiphilic polymer in various solvents.

\section{EXPERIMENTAL}

\section{Materials}

Commercially available sodium $p$-styrenesulfonate (SS) (Tokyo Kasei) was purified by recrystallization from ethanol-water mixed solvent. Butyl methacrylate (BMA) (Tokyo Kasei) was treated with $5 \% \mathrm{NaOH}$ solution to remove the inhibitor and dried over anhydrous sodium sulfate. $N$-Vinylcarbazole $(\mathrm{VCz})$ (Nakarai Chemicals) was recrystallized from ethanol. The preparation of viologens and $p$ cyano- $N$-methylpyridinium $\left(\mathrm{Py}^{+}\right)$quenchers is described elsewhere. ${ }^{1 \mathrm{~b}}$

\section{Preparation of Amphiphilic Polymer}

Terpolymerization of SS $(0.5 \mathrm{~g})$, BMA $(2.23 \mathrm{ml})$, and $\mathrm{VCz}(0.1 \mathrm{~g})$ was carried out with $2,2^{\prime}$-azobis(isobutyronitrile) (AIBN) initiator $(0.02 \mathrm{~g})$ in dimethylformamide (DMF)-water mixed solvent $\left(\mathrm{DMF} / \mathrm{H}_{2} \mathrm{O}=3 / 1\right)(60 \mathrm{ml})$. The polymerization solution was poured into a large quantity of water. The unreacted monomers and AIBN were extracted with ether. The polymer solution was filtrated with a $5-\mu \mathrm{m}$ membrane filter to remove macroaggregates. Finally the polymer was purified by ion exchanging with cation (Dowex 50W-X8) and anion (Amberlite IRA-40) exchange resins (the polymer solution was passed through the column packed with the resins), followed by ultrafiltration (Toyo ultrafilter UK-200). The composition of the polymer was determined by elemental analysis to be $4.8: 2: 1$ for BMA : SS : VCz.

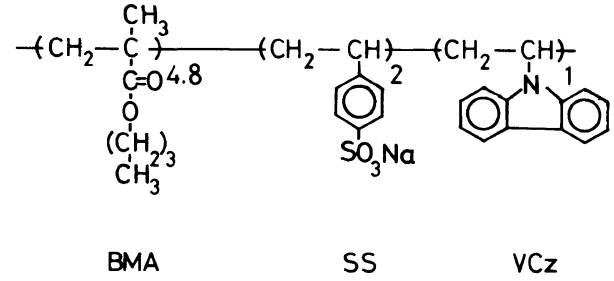

\section{Measurements}

The fluorescence spectrum was measured with a Shimadzu RF 503A spectrofluorophotometer. Polymer latex particles were observed with a Hitachi H-300 electron microscope. Flash photolysis for the detection of a transient viologen radical cation was carried out by a flash (pulse width: $10 \mu \mathrm{s}$ ) with an energy of $145 \mathrm{~J}$ from xenon-filled flash lamps. Wavelengths from $320 \mathrm{~nm}$ to $440 \mathrm{~nm}$ were used for the excitation of carbazole chromophore with cut-off filters (D-25 and UV-32, Toshiba).

\section{RESULTS AND DISCUSSION}

\section{Fluorescence Spectra}

The amphiphilic polymer was soluble in THF and DMF, whereas it dispersed in water as a latex particle. The diameter of the latex particle was estimated to be $200 \mathrm{~nm}$ from an electron micrograph. The fluorescence and excitation spectra of the carbazole covalently attached to the polymer were measured in various organic solvents and water. The structured fluorescence characteristic of monomeric carbazole chromophore with a strong $(0,0)$ band at about $350 \mathrm{~nm}^{7}$ was observed (Figure 1), and an excimer emission around $430 \mathrm{~nm}^{8}$ was not found with any appreciable intensity. These findings indicate that the carbazole chromophores are isolated from each other in the polymer. The shape and wavelength of the emission bands in the fluorescence spectra were unchanged with solvents. The emission intensity, however, varied with solvents. The fluorescence of the carbazole was the most intense in water and decreased in organic solvents. The decrease in the emission intensity 


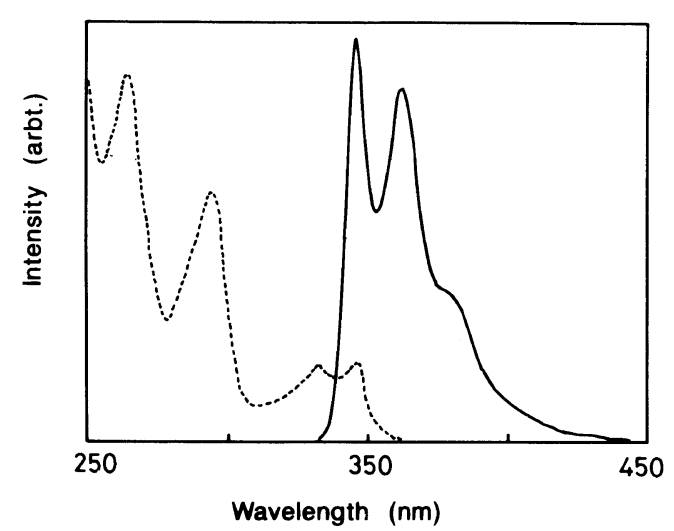

Figure 1. Emission and excitation spectra of carbazole covalently attached to amphiphilic polymer in water. - , emission $\left(\lambda_{\mathrm{ex}}=320 \mathrm{~nm}\right)$; ----, excitation $\left(\lambda_{\mathrm{em}}=\right.$ $365 \mathrm{~nm})$.

means that the deactivation of the excited carbazole is promoted in their solvents. Neither electron nor energy transfer quenching by the solvent molecules is possible from the viewpoint of energy and from no overlapping of acceptor-donor orbits. It has been reported that the fluorescences of aromatic series such as anthracene, pyrene, and triphenylene decrease by the thermal deactivation process based on charge transfer interaction with solvent molecules. ${ }^{9}$ We measured the fluorescence of ethylcarbazole in organic solvents to examine the interactions. The fluorescence intensity was unchanged by their solvents.

Figure 2 shows plots of intensity against the solubility parameters $(S)$ of solvents. The fluorescence intensity of the carbazole decreased with $S$. This parameter is a solvent parameter representing solvent polarity and defined as $S=(L / V)^{1 / 2}$, where $L$ is a molar vaporization energy and $V$ is molar volume. ${ }^{10}$ The solubility parameter is often used as a scale of the miscibility of polymers with solvents. The results may indicate that variation in emission intensity by solvent is related to the behavior of the polymer chain in solution. In THF or DMF, the polymer takes on an expanded form where energy migration between the carbazole units occurs along the

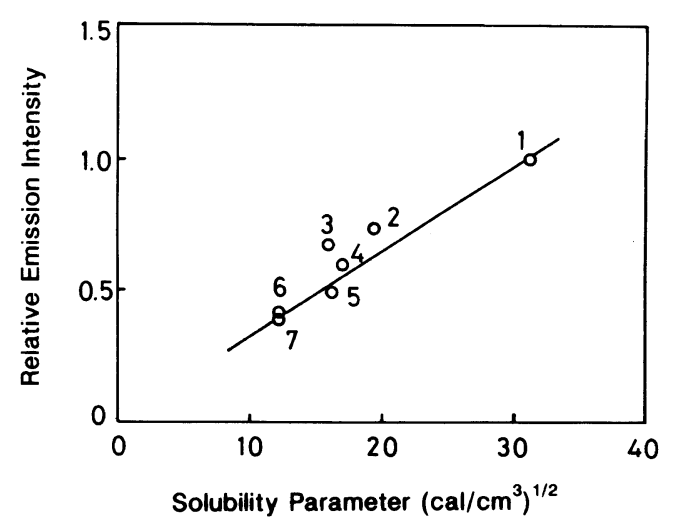

Figure 2. Plots of emission intensity of carbazole in various solvents versus the solubility parameter of the solvent. Intensity is relative ratio to that in water: 1 , water; 2, methanol; 3, ethanol; 4, acetonitrile; 5, DMF; 6 , THF; 7, ethylacetate.

polymer chain. If there is an energy trapping site in the polymer chain, the excitation energy is trapped at that site. As the energy trapping site, non-emissive excimer forming site or the carbazole unit interacting with anionic groups may be considered. We have, however, no spectroscopic evidence to clarify the site. In the latex particle of the polymers in water, the carbazole chromophores exist in their hydrophobic core and hydrophilic styrenesulfonate units are situated away from the carbazole groups. The hydrophobic aggregation favors interaction between the carbazole chromophores. If the energy trapping site exists in the hydrophobic core, the emission intensity should decrease in water. Therefore, the high fluorescence intensity in water suggests that the energy trapping site is based on the carbazole interacting with anionic groups, rather than the carbazole units in the hydrophobic core. Since the rotational relaxation process also influences emission intensity, ${ }^{11}$ the high intensity in water may be attributed to a slow rotational relaxation process due to the restricted movement of the carbazole unit in latex particles. The measurement of the rotational relaxation rate of carbazole unit in various solvents may be necessary for further dis- 
cussion.

\section{Fluorescence Quenching by Organic Cation} Quenchers

The fluorescence from the excited carbazole was quenched by various viologen (methyl $\left(\mathrm{MV}^{2+}\right)$, butyl $\left(\mathrm{BV}^{2+}\right)$, and benzyl $\left(\mathrm{BeV}^{2+}\right)$ substituents) and pyridinium $\left(\mathrm{Py}^{+}\right)$cations. The formation of methylviologen radical cation with the absorption maximum at $605 \mathrm{~nm}$ was confirmed by flash photolysis of a solution containing the polymer and viologen quencher, which suggests that the quenching proceeds by an electron transfer from the excited carbazole to the methylviologen quencher. We were not able to detect clearly transient absorption around $780 \mathrm{~nm}$ due to the carbazole cation radical. ${ }^{2 a}$ This may be attributed to some decomposition processes or reductive rapid electron transfer from the surrounding anionic species. The redox potential of the excited carbazole was estimated to be about $-2.74 \mathrm{~V}$ ( $v s$. SCE) from singlet excitation energy and the redox potential of carbazole. ${ }^{6}$ The more negative redox potential supports the electron transfer quenching mechanism; that is, the electron transfer from the excited carbazole to the viologen quencher is exothermic and a very favorable process.

Figure 3 shows plots of the ratios of fluorescence intensities in the absence and presence of the viologen quencher in water at various polymer concentrations. The plots deviated from a linear relationship expected from a Stern-Volmer quenching mechanism. The quenching efficiency changed by the polymer concentration; the fluorescence quenching became more effective as the polymer concentration was diluted with water. Since the viologen cation quencher should be associated with the anionic charged latex particle due to electrostatic and/or hydrophobic interactions, the effective quenching at a dilute polymer concentration in water can be explained by increase in the number of quencher molecules per latex particle (concentration effect). On the

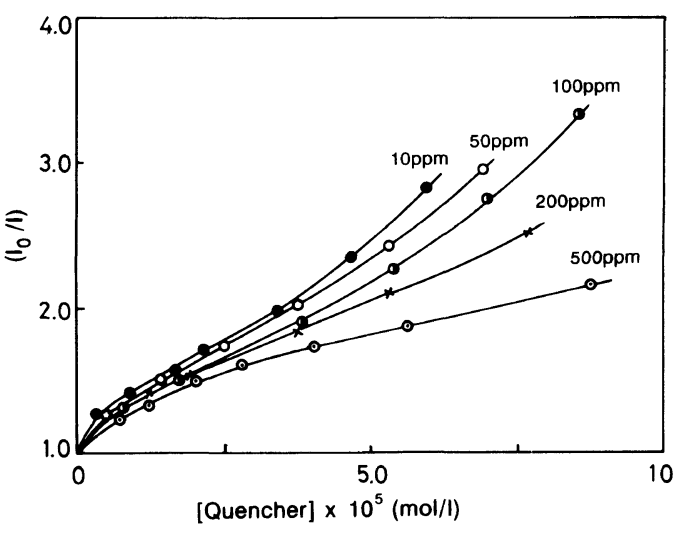

Figure 3. Change in emission intensity for quenching of carbazole by $\mathrm{BV}^{2+}$ quencher as a function of polymer concentration in water.

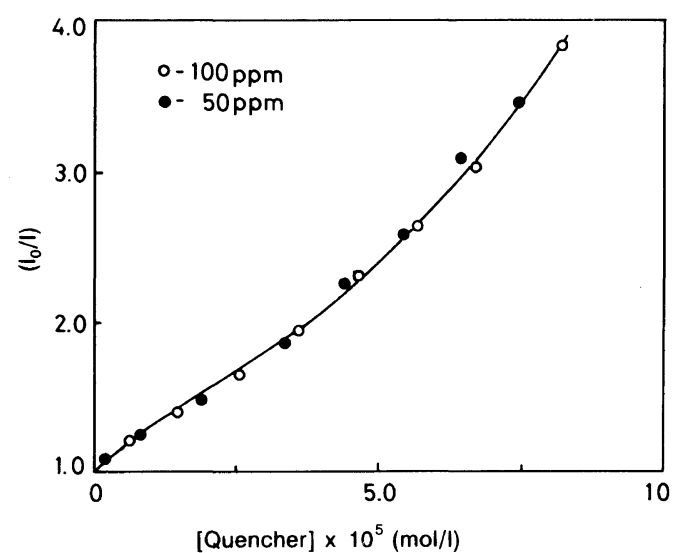

Figure 4. Plots for quenching of carbazole by $\mathrm{BV}^{2+}$ in DMF solution.

other hand, in DMF solution, where the polymer is soluble homogeneously, the change in quenching efficiency by polymer concentration was not observed (Figure 4).

The differences in quenching efficiency among various quenchers in water are shown in Figure 5. The viologen dication quenchers were more effective than the pyridinium monocation quencher. The redox potentials of $\mathrm{Py}^{+}$, $\mathrm{MV}^{2+}, \mathrm{BV}^{2+}$, and $\mathrm{BeV}^{2+}$, are $-0.60,-0.45$, -0.45 , and $-0.37 \mathrm{~V} v s$. SCE in acetonitrile, respectively. This order of quenching efficiency may be primarily explained by the redox potentials of the quenchers. $\mathbf{M V}^{2+}$ and $\mathbf{B V}^{2+}$ 


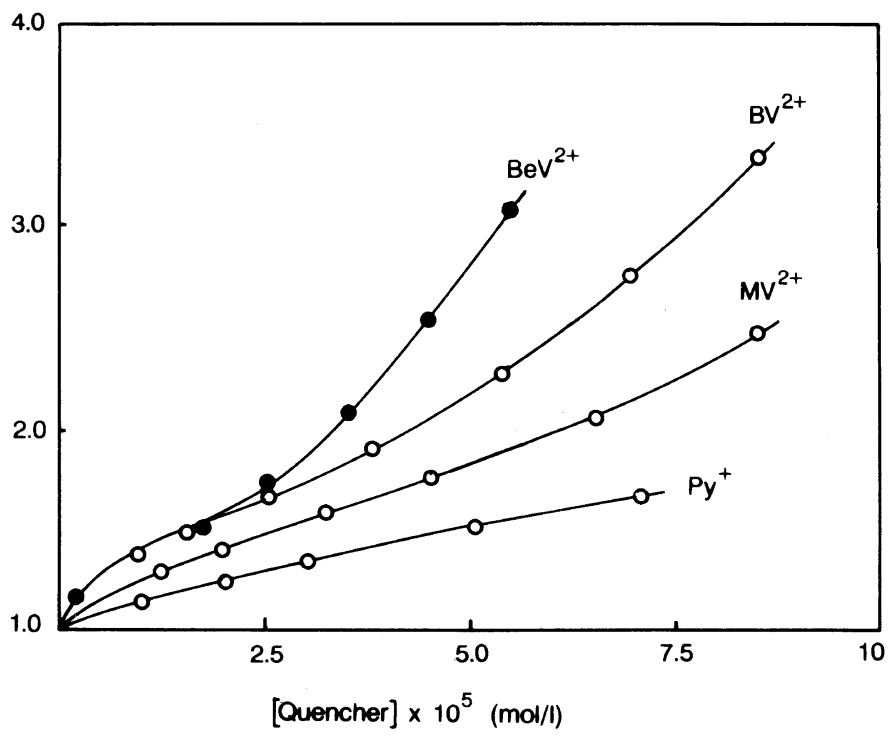

Figure 5. Plots for quenching of carbazole by various quenchers in water. Polymer concentration is 100 ppm.

have the same redox potential and showed the same efficiency in the quenching of ethylcarbazole and ruthenium complex ${ }^{1 \mathrm{~b}}$ in organic solvents. The difference in the quenching in water between $\mathrm{MV}^{2+}$ and $\mathrm{BV}^{2+}$ shown in Figure 5 is attributed to the difference in hydrophobic interactions. The quencher must enter the hydrophobic region to interact with the carbazole chromophore. The viologen with longer alkyl substituent becomes a more effective quencher in water. An increase in quenching efficiency by the incorporation effect due to hydrophobic interaction was already found in the previous work on emission quenching in a dispersion of a polymer latex. ${ }^{6}$ On the other hand, the hydrophobic interaction often acted as an unfavorable factor in a quenching reaction. In the quenching of photoexcited ruthenium complex with various viologen quenchers in SDS micellar solution, we reported that the quenching efficiency of viologens decreases with increasing alkyl chain length in the order methyl $>$ ethyl $>$ propyl $>$ butyl $>$ hexyl. ${ }^{1 \mathrm{~b}}$ The quenching reaction is SDS micellar solution proceeds on the surface

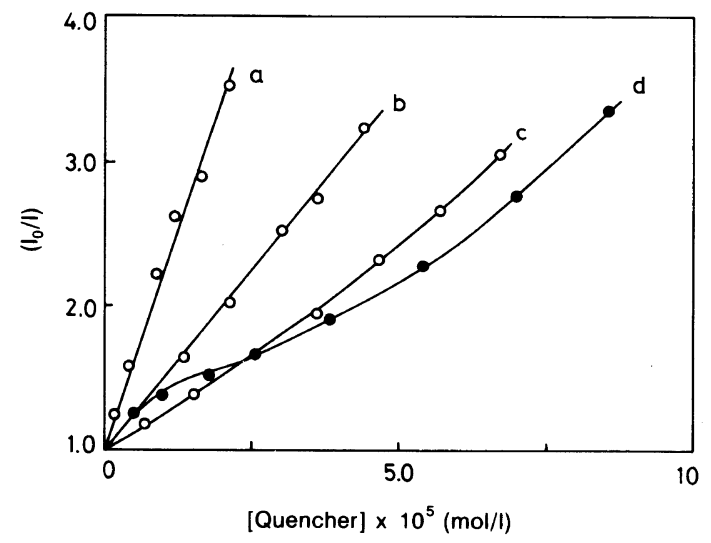

Figure 6. Plots of emission intensity of carbazole against concentration of $\mathrm{BV}^{2+}$ in various solutions. (a), aqueous THF (a few \% water); (b), a 1:1 mixture of THF.

region of the micelle, and determined by the surface diffusion of viologen. As the alkyl chain becomes longer, the viologen quenchers are bound tightly to the micelle due to hydrophobic interaction, which results in a lowering of quenching efficiency by the suppression of the surface diffusion of viologen. ${ }^{12}$ The present amphiphilic polymer 
forms a latex particle in water. The effect of alkyl substituents, therefore, shows a similar behavior with that in polymer latex reported previously.

Figure 6 shows plots for the quenching of carbazole by $\mathrm{BV}^{2+}$ in various solutions. The effective quenching in aqueous THF is attributed to the incorporation of the viologen quencher into the polymer as a counter cation of a contact ion-pair, because the viologen, added from an aqueous solution, is less soluble in aqueous THF. The addition of $\mathrm{H}_{2} \mathrm{O}$ (a mixture of THF and $\mathrm{H}_{2} \mathrm{O}$ ) decreases the quenching efficiency due to dissociation of the viologen from the polymer chain. Moreover, the polymer chain has an expanded form in THF and DMF and the carbazole chromophore is encountered effectively with the quencher. Plots for the quenching in THF, DMF, and a mixture of THF and $\mathrm{H}_{2} \mathrm{O}$ show the normal behavior often found in quenching for homogeneous systems. In a DMF solution, quenching efficiency is changed neither by polymer concentration nor alkyl chain length of the viologen, whereas change by these factors was observed in water as described above. The difference in the quenching behavior is caused by conformation of the polymer chain in solutions.

\section{REFERENCES}

1. a) J. K. Thomas and M. Almgren, "Solution Chemistry of Surfactants," Vol. 2, K. L. Mittal, Ed., Plenum Press, New York, N. Y., 1979, p 559; b) T. Miyashita, T. Murakata, and M. Matsuda, J. Phys. Chem., 87, 4529 (1983); c) T. Miyashita, T. Murakata, Y. Yamaguchi, and M. Matsuda, J. Phys. Chem., 89, 497 (1985).

2. a) Y. Yamaguchi, T. Miyashita, and M. Matsuda, $J$. Phys. Chem., 85, 1369 (1981); b) M. Almgren, F. Grieser, and J. K. Thomas, J. Am. Chem. Soc., 102, 3188 (1980).

3. T. Nomura, J. R. Escabi-Perez, J. Sunamoto, and J. H. Fendler, J. Am. Chem. Soc., 102, 1484 (1980).

4. a) Y. Morishima, T. Kobayashi, T. Furui, and S. Nozakura, Macromolecules, 20, 1707 (1987); b) T. Miyashita and M. Matsuda, Polym. Commun., 25, 138 (1984).

5) a) K-P. Seefeld, D. Mobius, and H. Kuhn, Helv. Chim. Acta, 60, 2608 (1977); b) T. Murakata, T. Miyashita, and M. Matsuda, J. Phys. Chem., 92, 6040 (1988).

6. T. Miyashita, M. Ohsawa, and M. Matsuda, Macromolecules, 19, 585 (1986).

7. G. E. Johnson, J. Phys. Chem., 78, 1512 (1974).

8. R. Solaro, G. Galli, A. Ledwith, and E. Chiellini, "Polymer Photophysics," D. Phillips, Ed., Chapman and Hall, New York, 1985, p 377.

9. D. K. Majumdar and S. Basu, J. Chem. Phys., 33, 1191 (1960).

10. J. Brandrup and E. H. Immergut, Eds., "Polymer Handbook," Wiley, New York, N. Y., 1975.

11. G. Oster and Y. Nishijima, J. Am. Chem. Soc., 78, 1581 (1956).

12. T. Miyashita, T. Murakata, and M. Matsuda, J. Phys. Chem., 93, 1426 (1989). 